Meta

Journal des traducteurs

Translators' Journal

\title{
Terminologie anglais-français des systèmes experts et des sujets connexes (suite)
}

\section{Jacques Lethuillier et Monique C. Cormier}

Volume 36, numéro 2-3, juin 1991

URI : https://id.erudit.org/iderudit/003489ar

DOI : https://doi.org/10.7202/003489ar

Aller au sommaire du numéro

Éditeur(s)

Les Presses de l'Université de Montréal

ISSN

0026-0452 (imprimé)

1492-1421 (numérique)

Découvrir la revue

Citer cet article

Lethuillier, J. \& Cormier, M. C. (1991). Terminologie anglais-français des systèmes experts et des sujets connexes (suite). Meta, 36(2-3), 490-497. https://doi.org/10.7202/003489ar d'utilisation que vous pouvez consulter en ligne. 


\section{ÉTUDES TERMINOLOGIQUES ET LINGUISTIQUES}

\section{TERMINOLOGIE ANGLAIS-FRANÇAIS DES SYSTÈMES EXPERTS ET DES SUJETS CONNEXES (SUITE, $c f$. META 35-4, pp. 748-759.)}

Ce lexique bilingue, non exhaustif, constitue une liste de travail à partir de laquelle est menée une étude méthodique de la terminologie des systèmes experts, qui débouchera sur la production d'un vocabulaire avec définitions. Une deuxième tranche, accompagnée d'un index anglais-français et d'une bibliographie, fera l'objet d'un autre article dans un prochain numéro de la revue.

parcelle de connaissance - Syn. de fragment de connaissance.

parcours d'un arbre recherche arborescente, exploration d'arbre

tree search

parcours en largeur - Syn. de parcours en largeur d'abord.

parcours en largeur d'abord parcours en largeur, exploration en largeur d'abord,

recherche en largeur d'abord

breadth-first search

pas de raisonnement - Syn. de inférence logique.

planificateur - Syn. de générateur de plans.

planification hiérarchique

hierarchical planning

top-down plan elaboration, plan refinement

poids - Syn. de arité.

$\mathrm{POO}$ - Syn. de programmation orientée objet.

portée d'une variable

scope (of a variable)

précondition - Syn. de prémisse.

préjugé

prejudice

prémisse

antécédent, condition, précondition

premise

if part, LHS, left-hand side, situation, condition, antecedent

pré-système - Syn. de maquette.

preuve

proof

logical deduction, logical proof

preuve de théorème

theorem proof

Meta, XXXVI, 2/3, I99I 
preuve par réfutation

preuve par contradiction

refutation proof

reductio ad absurdum, proof by contradiction

preuve par résolution

resolution proof

principe de circonscription - Syn. de circonscription.

principe de résolution - Syn. de résolution.

procédure alpha-bêta - Syn. de algorithme alpha-bêta.

procédure par réfutation

refutation procedure

procédure réflexe - Syn. de attachement procédural.

procédé alpha-bêta - Syn. de algorithme alpha-bêta.

production - Syn. de règle de production.

programmation en règles de production

rule-oriented programming

programmation logique

logic programming

programmation orientée objet

programmation par objet, $\mathrm{POO}$

object-oriented programming

OOP

programmation par objet - Syn. de programmation orientée objet.

programme de démonstration de théorèmes - Syn. de démonstrateur de théorème.

Prolog

Prolog

propagation de contraintes

propositional constraint propagation

proposition

proposition

simple proposition, atomic proposition

proposition composée

expression bien formée, FBF, formule bien formée, formule

logique composée, forme propositionnelle

propositional sentence, proposition, compound proposition, Well-Formed Formula, WFF

prototypage - Syn. de maquettage.

prototype - Syn. de maquette.

prototype

unité, frame, forme-objet, cadre, schéma

frame

unit, object

quantificateur

quantifier 
quantificateur existentiel existential quantifier

quantificateur universel universal quantifier

racine

$$
\text { tête }
$$

root node

root, top node

raisonnement

inférence

reasoning inferencing

raisonnement analogique - Syn. de raisonnement par analogie.

raisonnement de bon sens - Syn. de raisonnement de sens commun.

raisonnement de sens commun

raisonnement de bon sens

everyday reasoning

raisonnement déductif — Syn. de chaînage avant.

raisonnement hypothétique

hypothetical reasoning assumption-based reasoning

raisonnement incertain raisonnement sur l'incertain inexact reasoning raisonnement inductif - Syn. de induction.

raisonnement inductif — Syn. de chaînage arrière.

raisonnement non monotone raisonnement révisable

nonmonotonic reasoning

raisonnement par analogie raisonnement analogique

analogical reasoning

raisonnement par défaut

default reasoning

raisonnement par l'absurde — Syn. de modus tollens.

raisonnement régressif - Syn. de chaînage arrière.

raisonnement révisable - Syn. de raisonnement non monotone.

raisonnement sur l'incertain — Syn. de raisonnement incertain.

raisonnement temporel

temporal reasoning

$\mathrm{RCO}$ - Syn. de représentation par objets.

recherche aléatoire - Syn. de recherche aveugle.

recherche arborescente - Syn. de parcours d'un arbre. 
recherche avec retour arrière retour arrière

backtracking backtrack

recherche aveugle recherche aléatoire, stratégie sans information, recherche sans information blind search uninformed search

recherche bidirectionnelle bidirectional search

recherche en graphe graph search

recherche en largeur d'abord - Syn. de parcours en largeur d'abord.

recherche en profondeur

depth-first search

recherche exhaustive

exhaustive search

recherche heuristique recherche informée

heuristic search heuristically guided search, directed search, best first search

recherche informée - Syn. de recherche heuristique.

recherche sans information - Syn. de recherche aveugle.

registre de mémoire à court terme — Syn. de base de faits.

règle

rule

règle applicable

candidate rule

règle avec variable

variable rule

règle d'inférence

règle de dérivation, règle de déduction, règle déductive inference rule

règle d'introduction

introduction rule

règle de déduction - Syn. de règle d'inférence.

règle de défaut - Syn. de défaut.

règle de dérivation - Syn. de règle d'inférence.

règle de détachement — Syn. de modus ponens.

règle de production production

production rule production, IF-THEN rule 
règle de réécriture

rewrite rule

règle de stratégie métarègle, stratégie de contrôle metarule

règle de substitution uniforme - Syn. de substitution uniforme.

règle déductive - Syn. de règle d'inférence.

règle experte

knowledge rule

règle incertaine

uncertain rule

règle par défaut - Syn. de défaut.

représentation centrée objet - Syn. de représentation par objets.

représentation de connaissance centrée objet - Syn. de représentation par objets.

représentation de connaissance orientée objet - Syn. de représentation par objets.

représentation de la connaissance - Syn. de représentation des connaissances.

représentation des connaissances

représentation de la connaissance

knowledge representation

représentation déclarative

declarative representation

représentation en objets - Syn. de représentation par objets.

représentation logique (des connaissances)

logic-based representation

logical representation, logical knowledge representation

représentation objet - Syn. de représentation par objets.

représentation par objets

représentation objet, représentation centrée objet,

représentation de connaissance orientée objet, $\mathrm{RCO}$,

représentation de connaissance centrée objet,

représentation en objets, représentation par objets structurés

object-oriented knowledge representation

représentation par objets structurés - Syn. de représentation par objets.

représentation procédurale

procedural representation

représentation réseau

network representation

réseau - Syn. de graphe.

réseau sémantique

semantic network semantic net

résolution

méthode de résolution, principe de résolution 
resolution inference rule resolution principle

résolution de conflits

conflict resolution

résolution de problèmes

problem solving

résolution non clausale

non-clausal resolution

$\mathrm{NC}$ resolution

résolvant - Syn. de clause résolvante.

résolvante - Syn. de clause résolvante.

retour arrière - Syn. de recherche avec retour arrière.

rubrique - Syn. de case.

SAC - Syn. de système d'acquisition des connaissances.

savoir - Syn. de connaissance.

SC - Syn. de source de connaissance.

scénario - Syn. de script.

schéma - Syn. de prototype.

script

scénario

script

$\mathrm{SF}$ - Syn. de système axiomatique.

si et seulement si

iff

if and only if

SIAD - Syn. de système interactif d'aide à la décision.

SIAD

Système Interactif d'Aide à la Décision

DSS

Decision Support System

sommet - Syn. de nœud.

source de connaissance

$\mathrm{SC}$

knowledge source

spécialisation universelle

universal specialization

stratégie d'implication minimale - Syn. de implication minimale.

stratégie de contrôle - Syn. de règle de stratégie.

stratégie de moindre engagement - Syn. de implication minimale.

stratégie de raisonnement

reasoning strategy

stratégie du donné

linear input resolution 
stratégie du gradient

hill climbing

stratégie du support

set-of-support strategy

stratégie sans information - Syn. de recherche aveugle.

stratégie unité

unit resulting resolution

substitution

substitution

substitution uniforme

règle de substitution uniforme

consistent substitution

syllogisme

syllogism

système à base de connaissances

knowledge-based system

système à base de règles de production — Syn. de système à règles de production.

système à règles de production

système expert à règles de production, système de production,

système à base de règles de production

rule-based system

shallow reasoning system, production system

système à tableau noir

blackboard system

système axiomatique

axiomatique, $\mathrm{SF}$, système formel

axiom system

formal deduction system, FS, formal system

système d'acquisition des connaissances

SAC

knowledge input subsystem

knowledge acquisition subsystem

système de production — Syn. de système à règles de production.

système expert

SE

expert system

système expert à règles de production - Syn. de système à règles de production.

système formel - Syn. de système axiomatique.

Système Interactif d'Aide à la Décision - Syn. de SIAD.

table de vérité

truth table

tableau noir

blackboard

terme 


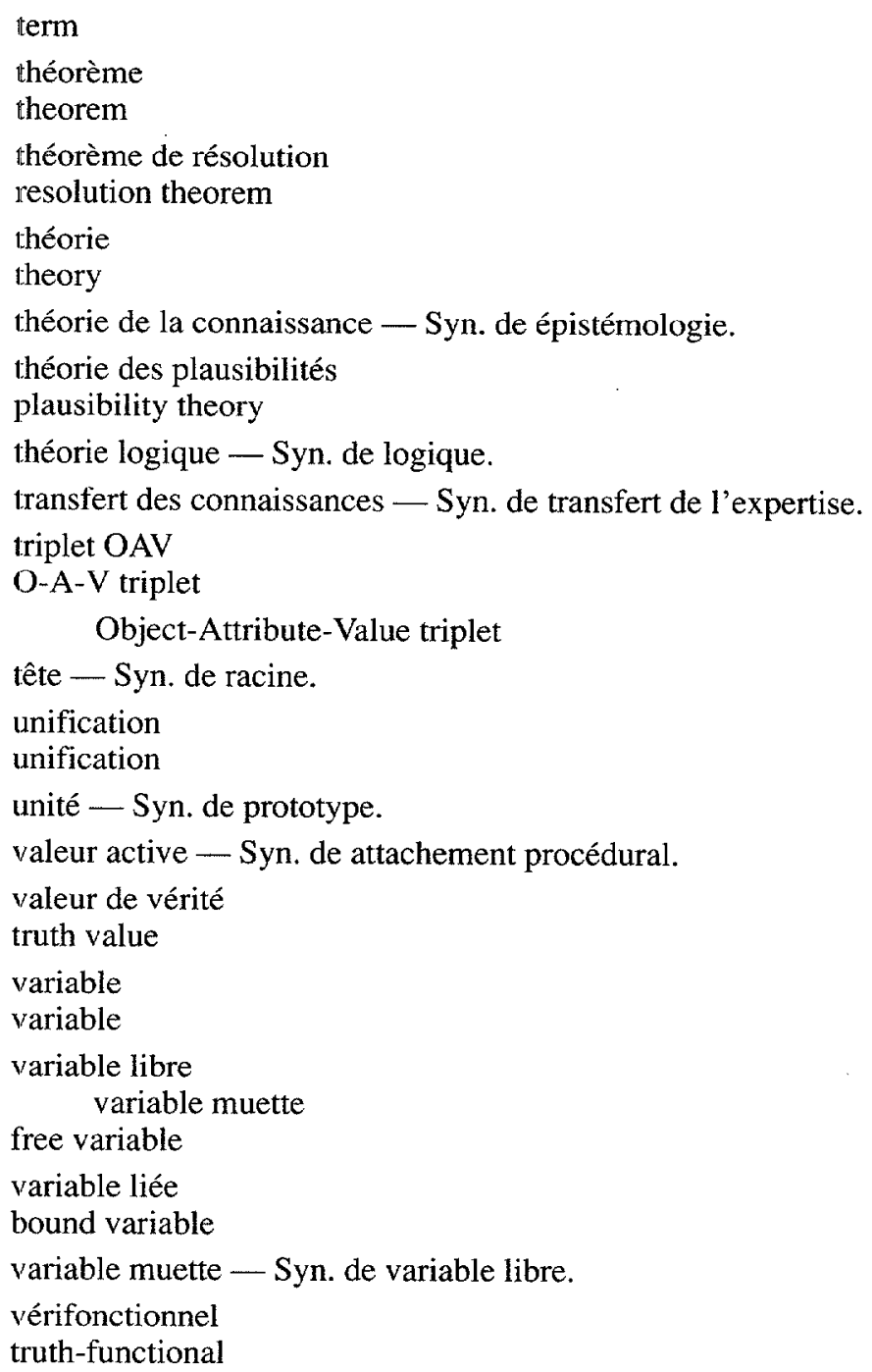

